\title{
Influential Factors of Emotional Display Rules in Chinese Adolescents
}

\author{
Yifang Wang", Xue Liu, Lixia Cui \\ Beijing Key Laboratory of Learning and Cognition, Department of Psychology, \\ Capital Normal University, Beijing, China \\ Email: "wangyifang6275@gmail.com
}

Received March 16 ${ }^{\text {th }}$, 2012; revised April $9^{\text {th }}$, 2012; accepted May $11^{\text {th }}, 2012$

\begin{abstract}
To explore the influential factors of emotional display rules in Chinese adolescents, 119 participants (male 66, female 53) were selected from a junior high school in Beijing. Ten life events associated with emotional display rules were used to comprehensively examine the effects of emotional type (positive/ negative), interactive partners (parents/peers), other-involved/self-involved and gender. Results indicated that in the positive contexts, adolescents applied more EDR in front of peers than parents, while in the negative contexts their performances were similar in front of the two kinds of interactive partners. All the participants used more EDR in negative contexts than positive contexts. Compared with other-involved situations, they applied more EDR in self-involved situations. Girls used more EDR than boys.
\end{abstract}

Keywords: Adolescents; Emotional Display Rules; Emotional Types; Interactive Partners; Other-/Self/Involved; Gender

\section{Introduction}

The expression of emotion is likely to vary depending on social expectation in specific social environment, which is called "emotional display rules (EDR)" proposed firstly by Ekman and Friesen (See Hou \& Yu, 2006). The findings indicated that there was a good relationship between individuals' ability to apply EDR and social abilities (Jones, Abbey, \& Cumber 1998; McDowell \& Parke, 2000). Underwood (1992) found that compared with non-aggressive primary students, aggressive individuals used less EDR to disguise their anger.

The application of EDR started from preschool age and rapidly developed during primary school age. Cole (1986) found through natural observation that 4 year-old girls could apply EDR in upset situations. The findings of Shipman and Zeman (2001) indicated that 6 year-old children were skilled at distinguishing facial expressions and inner experiences. The individuals' knowledge on EDR increased from age 6 to 10 (Gnepp \& Hess, 1986). Underwood (1992) also demonstrated that 10 year-olds performed better than 8 year-olds on regulating outer emotional expressions, but there were no differences between 10 year-olds and 13 year-olds. These results suggested that individuals' knowledge of EDR increased with age and stabilized in high grade of primary school.

The development of EDR was influenced by many factors including gender, emotional types and interactive partners. Many researchers found that girls' ability to apply EDR was better than boys of the same age (Underwood, Coie, \& Herbsman, 1992; Jones, Abbey, \& Cumberl, 1998; Garrett-Peters \& Fox, 2007). Boys expressed more negative emotions when receiving unflavored presents, while girls expressed more positive emo-

*This research was supported by Project of National Natural Science Foundation of China [30900407 to Yifang Wang], Project of Humanities and Social Science Fund of Chinese Education Ministry [09YJCXLX023 to Yifang Wang and Project of Beijing Government [PHR201007109 to Ping Fang].

${ }^{\#}$ Corresponding author. tions (Saarni, 1984). However, some researches demonstrated no gender differences (Gnepp \& Hess, 1986; McDowell \& Parke, 2000).

The types of emotion included positive and negative ones. Most researchers focused on children's application of EDR in negative emotional contexts (Saarni, 1984; Zeman \& Garber, 1996; Garrett-Peters \& Fox, 2007). However, in real life, sometimes people need disguising their positive emotions. Harris (1986) compared the performances of children on EDR in both positive and negative emotional contexts, and found that children applied EDR more frequently in negative contexts. However, the findings of McDowell and Parke (2000) demonstrated no differences existed between the types of emotional contexts.

Children's applying EDR varied with different interactive partners. Underwood (1992) demonstrated that children were more likely to disguise their angers facing teachers than peers. Moreover, children adopted more emotional regulating behaviors facing peers than parents (Zeman \& Garber, 1996). Besides, the application of EDR included self-involved and otherinvolved situations. Individuals were more sensitive to emotions in self-involved situations (Wang \& Su, 2008).

According to the findings of previous studies, children acquired the knowledge of EDR at the age of 10. So adolescents possessed a good ability of EDR. Then whether the performances of adolescents on EDR were still influenced by those factors was in doubt. The present research aimed at exploring comprehensively the effects of these factors: gender, emotion types (positive/negative), interactive partners (parents/peers) and self-involved or other-involved situations on adolescents' EDR.

\section{Methods and Procedures}

\section{Participants}

The participants consisted of 119 students from a junior mi- 
ddle school in Beijing (66 male, 53 female). One hundred and sixteen students completed the measurement. The percentage of validity was $97.48 \%$.

\section{Measures}

We collected life events in adolescent period by interviews and literature references. Ten typical life events including 5 positive and 5 negative emotional contexts associated with EDR were selected and rewritten according to Josephs (1994) and Jones, Abbey, and Cumberl (1998). Because of different interactive partners (parents/peers) and other-involved or selfinvolved situations, 10 typical contexts generated 40 situations associated with EDR.

For example, Lin Ming's birthday is coming. His dream present from parents is a car model. However, he receives a present which he does not like. What will Lin Ming do?

A. display sad facial expression

B. feel sad, but display calm facial expression

C. feel sad, but display happy facial expression

D. others

If you were him, what would you do?

A. display sad facial expression

B. feel sad, but display calm facial expression

C. feel sad, but display happy facial expression

D. others

If participants chose A, it represented that they didn't use EDR. If they chose B or C, it represented their use of EDR. Participants who chose D were encoded into two categories by two undergraduates majoring in psychology: participants with the use of EDR or not. The kappa was $.87^{* * *}$. Using EDR scored 1, while not using EDR scored 0.

\section{Results}

The performance of applying emotional display rules in adolescents was shown in Table 1. A 2 (emotional types) $\times 2$ (interactive partners) $\times 2$ (other-involved/self-involved) $\times 2$ (gender) repeated measure ANOVA was conducted. It yielded significant interactions among emotional types, interactive partners, and other-involved/self-involved situations, $F(1,114)=8.33, p$ $<.01, \eta^{2}=.07$, and between emotional types and interactive partners, $F(1,114)=8.52, p<.01, \eta^{2}=.07$. It also yielded significant main effects of emotional types, $F(1,114)=188.53$, $p<.01, \eta^{2}=.62$, interactive partners, $F(1,114)=5.85, p<.05$, $\eta^{2}=.05$, other-involved/ self-involved situations, $F(1,114)=$ 13.97, $p<.01, \eta^{2}=.11$, and gender, $F(1,114)=10.74, p<.01$, $\eta^{2}=.09$.

Simple effect analysis was done to explain the significant interactions among emotion types, interactive partners and selfinvolved or other-involved situations. In positive emotional cotexts, participants applied more EDR in front of peers than parents in self-involved situation, $t(115)=-3.40, p<.01$, and in other-involved situation, $t(115)=-2.16, p<.05$. While in negative emotional contexts, no significant differences existed between two kinds of interactive partners both in self-involved situations, $t(115)=1.19, p>.05$, and in other-involved situations, $t(115)=-1.43, p>.05$.

\section{Discussion}

In the positive contexts, adolescents applied more EDR in front of peers than parents, while in negative contexts, their
Table 1.

The performance of applying emotional display rules in adolescents $(M(S D))$.

\begin{tabular}{cccccc}
\hline \multirow{2}{*}{ Gender } & \multirow{2}{*}{ Emotion } & \multicolumn{2}{c}{ Parents } & \multicolumn{2}{c}{ Peers } \\
\cline { 3 - 6 } & & Other $(0$ - 5) & Self $(0$ - 5) & Other $(0$ - 5) & Self $(0$ - 5) \\
\hline \multirow{2}{*}{$\mathrm{B}$} & $\mathrm{N}$ & $3.30(1.34)$ & $3.80(1.29)$ & $3.12(1.54)$ & $4.01(1.12)$ \\
& $\mathrm{P}$ & $1.62(1.53)$ & $2.19(1.52)$ & $1.83(1.49)$ & $2.28(1.49)$ \\
& $\mathrm{N}$ & $4.17(1.05)$ & $4.24(1.01)$ & $4.12(1.21)$ & $4.25(1.11)$ \\
$\mathrm{H}$ & $\mathrm{P}$ & $1.86(1.56)$ & $2.29(1.74)$ & $2.49(1.38)$ & $2.78(1.57)$ \\
\hline
\end{tabular}

performances were similar in front of the two kinds of interactive partners. All the participants used more EDR in negative contexts than in positive contexts. Compared with other-involved situations, they applied more EDR in self-involved situations. Girls used more EDR than boys.

The results indicated that the effects of interactive partners on adolescent's application of EDR were moderated by emotional types. Zeman and Garber (1996) found individuals' emotional expressions were influenced by degree of familiarity and sense of subordinating to elders or superiors. Although peers are increasingly important to individuals in early adolescence, the high familiarity and subordinate sense with their parents can still not go beyond. Adolescents and their parents are the community of interests. When faced with positive events, they are unconsciously willing to share with each other. However, peers are different from parents though adolescents spend more time with peers. Establishment and maintenance of peer relationship is an important life event for them. To get a better peer relationship, sometimes they disguise their positive emotions in front of peers in order to put little pressure on them. Take a scenario for example. "In an athlete meeting, Lin Min won the first prize, while his friends got nothing." Lin Min would try to apply EDR to disguise their positive emotions perhaps because he worried about giving his peers too much pressures or get peers jealous of him.

Zeman and Garber found that in angry and sad contexts, primary students applied more EDR in front of peers than parents, which was inconsistent with our results. Liu and Fang (2007) demonstrated that 4 - 6 year-old children had a better understanding of situations interacting with peers than old generations, regardless of the types of emotions. Perhaps the different ages of participants accounted for the inconsistency of various findings. Seidel et al. (2010) indicated that happiness positively correlated with approaching intendancy, while anger positively correlated with avoidance intendancy. Individuals applied EDR in order to communicate with others more effectively. Adolescents expected receiving "respects like an adult" and smooth interpersonal relationship, so they were more likely to express positive feelings as well as deducting negative feelings.

Consistent with previous findings, we also found that compared with boys, girls were more likely to apply EDR. It could be of large possibility due to socialization of different gender roles. In most cultures, parents had different social expectations towards boys and girls. Girls should be more quiet and considerate. Thus, parents tolerated more boys' emotional expressions and asked girls to control their emotions. Cole (2005) suggested that parents focused more on girls' obedient emotions and boy's discordant emotions. The degree of parents' selective 
concentrations on specific emotions was related to children's later expression of specific emotions.

The results indicated that participants applied more EDR in self-involved than other-involved situations. Wang and Su (2008) demonstrated that individuals' emotional experiences were more sensitive in self-involved situations. Self-served biased attribution existed widely in population of different cultures (Mezulis, Abramson, Hyde, \& Hankin, 2004). Hence, adolescents made the judgments with different standards in selfinvolved and other-involved situations. In self-involved situations, they would protect themselves, while in other-involved situation, they would become more objective.

\section{REFERENCES}

Chaplin, T. M., Cole, P. M., \& Zahn-Waxler, C. (2005). Parental socialization of emotion expression: Gender differences and relations to child adjustment. Emotion, 5, 80-88. doi:10.1037/1528-3542.5.1.80

Cole, P. M. (1986). Children's spontaneous control of facial expression. Child Development, 57, 1309-1321. doi:10.2307/1130411

Garrett-Peters, P. T., \& Fox, N. A. (2007). Cross-cultural differences in children's emotional reactions to a disappointing situation. International Journal of Behavioral Development, 31, 161-169. doi:10.1177/0165025407074627

Gnepp, J., \& Hess, D. L. (1986). Children's understanding of verbal and facial display rules. Developmental Psychology, 22, 103-108. doi:10.1037/0012-1649.22.1.103

Harris, P. L., Donnelly, K., Guz, G. R., \& Pitt-Watson, R. (1986). Children's understanding of the distinction between real and apparent emotion. Child Development, 57, 895-909. doi:10.2307/1130366

Hou, R. H., \& Yu, G. L. (2006). Children's understanding of emotional display rules and use of strategies. Psychological Science (in Chinese), 29, 18-21.

Jones, D. C., Abbey, B. B., \& Cumberl, A. (1998). The Development of display rule knowledge: Linkages with family expressiveness and social competence. Child Development, 69, 1209-1222.

Josephs, I. E. (1994). Display rule behavior and understanding in preschool children. Journal of Nonverbal Behavior, 18, 301-326. doi:10.1007/BF02172291

Liu, Y. J., \& Fang, F. X. (2007). Research on the development of understanding of the situation using emotion display rules in 4- to 6year-old children (in Chinese). Chinese Journal of Behavioral Medical Science (in Chinese), 16, 241-243.

McDowell, D. J., \& Parke, R. D. (2000). Differential knowledge of display rules for positive and negative emotions: Influences from parents, influences on peers. Social Development, 9, 415-432. doi:10.1111/1467-9507.00136

Mezulis, A. H., Abramson, L. Y., Hyde, J. S., \& Hankin, B. L. (2004). Is there a universal positivity bias in attribution? A meta-analytic review of individual, developmental, and cultural differences in the self-serving attributional bias. Psychological Bulletin, 130, 711-747. doi:10.1037/0033-2909.130.5.711

Saarni, C. (1984). An observational study of children's attempts to monitor their expressive behavior. Child Development, 55, 15041513. doi: $10.2307 / 1130020$

Seidel, E. M., Ute, H., \& Michaela, K. (2010). The impact of facial emotional expressions on behavioral tendencies in women and men. Journal of Experimental Psychology: Human Perception and Performance, 36, 500-507. doi:10.1037/a0018169

Shipman, K., \& Zeman, J. (2001). Socialization of children's emotion regulation in mother-child dyads: A developmental psychopathology perspective. Developmental psychology and Psychopathology, 13, 317-336.

Underwood, M. K., Coie, J. D., \& Herbsman, C. R. (1992). Display rules for anger and aggression in school-age children. Child Development, 63, 366-380. doi:10.2307/1131485

Wang, Y. F., \& Su, Y. J. (2008). The developmental characteristics of 5 8-year-olds' faux pas detection and understanding. Psychological Science, 31, 324-327.

Zeman, J. \& Garber, J. (1996). Display rules for anger, sadness, and pain: It depends on who is watching. Child Development, 67, 957973. doi:10.2307/1131873 